\title{
Perceiving the Gap: Asynchronous Coordination of Plural Algorithms and Disconnected Logical Types in Ambient Space
}

\author{
Miki Goan ${ }^{1}$, Katsuyoshi Tsujita ${ }^{2}$, Takuma Ishikawa ${ }^{3}$, Shinichi Takashima ${ }^{4}$, \\ Susumu Kihara ${ }^{4}$, and Kenjiro Okazaki ${ }^{4}$ \\ 1 Osaka City University, \\ 3-3-138 Sugimoto Sumiyoshi-ku, Osaka 558-8585, Japan \\ goan@kmu-f.jp \\ 2 Osaka Institute of Technology, \\ 5-16-1 Ohmiya, Asahi-ku, Osaka 535-8585, Japan \\ tsujita@bme.oit.ac.jp \\ 3 Ochabi Institute, \\ 2-3 Kanda-surugadai, Chiyoda-ku, Tokyo 101-0062, Japan \\ takumaro2001@hotmail.com \\ 4 Kinki University, International Center for Human Sciences Yotsuya Art Studium, \\ Yotsuya 1-5, Shinjuku-ku, Tokyo 160-0004, Japan \\ \{shintakshintak, susumu.kihara, okazakipark\}@gmail.com
}

\begin{abstract}
The purpose of this study is to elaborate on the concept of "ambient space," the space which surrounds and moves with the self. For this purpose, this study focuses on the learning processes of the creation and viewing of art, related to both artists and viewers. Furthermore, we intend to deepen thought about ambient space and reconsider the dichotomy of "figure and ground." Through a workshop at an art museum, we showed that by stepping up from the logical type of figure-ground reversal perception - the figure-figure switch - the ground-ground switch, the perception of surfaces' layouts, can be perceived.
\end{abstract}

\section{A Step Up in Logical Type}

When we see evidence of distinguished skills, even if we cannot explain the excellence of the skill in words we can still be aware of it. Bateson's learning theory discusses the essential dilemmas and difficulties related to skill learning and the transmission of skills. As Bateson pointed out, one dilemma faced by artists is about a double effect of practice: "It makes him, on the one hand, more able to do whatever it is he is attempting; and, on the other hand, by the phenomenon of habit formation, it makes him less aware of how he does it" (Bateson, 1972, p.138). Another dilemma is that "if his attempt is to communicate about the unconscious components of his performance, then it follows that he is on a sort of moving stairway (or escalator) about whose position he is trying to communicate but whose movement is itself a function of his efforts to communicate" (op. cit., p. 138). Bateson deeply understood "that consciousness is necessarily

Y. Suzuki and T. Nakagaki (Eds.): WSH 2011 and IWNC 2012, PICT 6, pp. 130-147, 2013.

(C) The Author(s) 2013 
selective and partial, i.e., that the content of consciousness is, at best, a small part of truth about the self" (op. cit., p. 144). However, we agree with Bateson that the unconscious, as postulated by Freud, is not essentially unknowable. The framework he proposed assumed the existence of meta-level contexts (an invisible frame) that cover the algorithms in the area of so-called unconsciousness. In other words, the framework assumes the existence of things that enable measurement and judgment only as a function of logical typing. Bateson was a key figure in pointing out the reliance on the invisible and unconscious meta-frame and the great belief in the capability to step up logical types when we are in a dead-end situation, such as a double bind or an antinomy. He clearly pointed out this belief they can be a condition for learning.

\section{Ambient Space}

The purpose of this study is to elaborate on the concept of "ambient space," the importance of which was emphasized not only by G. Bateson but also by ecological psychologist J. J. Gibson and the painter J. Pollock, who developed their ecological views at around the same time. Ambient space refers to the space which surrounds and moves with the self. Bateson would say that it was the invisible context which is organised by logical types which can be recombined in several ways. For Gibson, it would be an environment whose structure would be specified by "ambient optic arrays," which refers to ambient light with structure. Ambient light means that "light would come to every point; it would surround every point; it would be environing at every point" (Gibson, 1979, p. 51). Light fills in so as to illuminate the structure of the environment, scattering and running against the myriad surfaces of objects in ambient space. "This implies an arrangement of some sort, that is, a pattern, a texture, or a configuration" (op. cit., p. 51). In Gibson's ground theory (1950), "space was no longer described as a void containing detached objects, but rather as intersecting surfaces; space was structurally more complex and differently organized" (Lombardo, 1987, p. 24). That is, Gibson denied Newton's concept of empty space, which declared that "the physical spatial world consisted of "objects" (static, self-contained things) possessing size, shape and location within space (a void)" (op. cit., p. 24). In ambient space, when we think of the relation between self and the ambient light which surrounds it, for example, it is necessary not to think first of the "things" which are related (the "relata") such as "self" and "light", but rather the opposite, so "the relations are to be thought of as somehow primary, the relata as secondary." "Beyond this, it is claimed that the relations are of the sort generated by processes of information exchange" (op. cit., p.154). Additionally, Lombardo (1987) indicated that Gibson's ecological concept of the ambient optic array (which is that reflected light diverges outward into the medium, but at any given point in the medium, light is converging from surrounding (ambient) objects) is very similar to Leibnitz's "monad": 
described as the embodiment of the surrounding whole within the part, an entity "mirrors" its relationships to all surrounding entities. The monad provided an alternative building block to Newton's atom, offering a relational view of reality. Instead of intrinsically independent atoms (elements), each of which is internally simple, the relational view rejects the idea of absolute parts independent of the whole" (op. cit., p. 52).

The artist Jackson Pollock expended great mental energy and strength to describe the ambient space. As a learner, Pollock analyzed the ambient space surrounding his body, invented painting techniques such as dripping and pouring, and attempted to reconstruct the ambient space on the canvas, the medium itself. However, generally speaking, describing and analyzing the ambient space is difficult. Although the idea of the ambient space that Gibson defined and Pollock demonstrated denies explanation by way of an analogy between the retinal image and the picture by using the pictorial model, regrettably even paintings by Pollock, who studied ambient space, are treated as finished products for image analysis. With all methods of fractal analyses for complex systems (Taylor et al., 2000), the idea that many hierarchical layers of arbitrary shapes are regarded as "ground" and the segments of "lines" intersecting hierarchical blocks, on the other hand, are regarded as "figures" is nothing but a tenet of image analysis. In the pictorial model, optical stimulation is regarded as having properties of momentarily fixed conditions; that is, a series of separate static pictures. However, the medium filled with light in ambient space does not form into an "image." Lombardo (1987, p. 39) explained that

Aristotle sees the medium as revealing forms rather than transmitting something material (eidola). The medium is a field actualized through light. It is not a void or an empty space - it possesses the power of "transparency". The perceiver sees forms through the medium; the perceiver does not see the medium... as Gibson would say, the perceiver does not see "light" — the perceiver sees by means of light.

\section{Focus on the Creation Process (Learning Process) of the Arts: Reconsideration of the Dichotomy of "Figure and Ground"}

Art is commonly concerned with learning, i.e., with bridging the gap between the more or less unconscious premises acquired by learning and the more episodic content of consciousness and immediate action (Bateson, 1972, p.308). This study focuses on the learning processes of creation and viewing, related to both artists and viewers, in order to elaborate the idea of ambient space. As an example, imagine that someone is asked to remember the assignment of white and black go stones on a go board after the final move? It is very difficult to guess correctly whether a stone is white or black by tracing the position on the go board from one corner in turns. It also has a high usage of memory resources. 
There is an easier way which is well known to the people learning to play go. This is simply to recall each move from the beginning in turns, and to assign the go stones to positions based on the moves. This study focuses on such a learning process. The learning process which is the significant focus of this study involves re-experiencing our own and others' learning processes. The learner need not repeat any previous misunderstandings or settle for the top-down lectures of specialists such as art historians or art critics, which are external to personal memories and experiences.

Another aim of this study is to deepen thought about ambient space, reconsidering the dichotomy of "figure and ground," which Gestalt psychology proposed. Considering a space as "the ambient space" that is filled with medium of the ambient optic array, the binary division into either figure or ground will be revealed to be insufficient to express such a space (Gibson, 1979). It is usually considered that "ground" is the expression of medium and is behind the "figure," the expression of the object. However, according to the definition of ambient space, the medium surrounds the object not only behind but also to the fore, that is, the whole. When seen from the perspective of ambient space, the idea of "figure-ground reversal" (Rubin, 1915) becomes insignificant because the idea is nothing but to return to a superficial viewing of difference, keeping the propriety claim of the dichotomy. But we don't intend to completely deny the dichotomy of figure and ground in the expression of space, insisting that it is too rough and of no use. We would like to verify the problem of "figure and ground" in a framework of stepping up the logical types as Bateson discussed. We did so through a workshop at an art museum held in a demonstrative manner. The details are described in the next section.

\section{Workshop in an Art Museum}

In this study, we would like to regard the arts as the imitation techniques used to identify the structure of the ambient space. Which is to say that it is not a problem of the technical transfer of skills acquired in one context to another. For example, studying painting will not lead to the making of a robot that can paint. However, creating a painting might utilize a modelling technique that has the same action level with producing robots or dramas, in terms of the modelling of the world.

This study accentuates the way of interdisciplinary research. Usually, as Bateson pointed out, interdisciplinary research occurs in a system in which, for example, an ecologist will need a geologist to tell him about the rocks and soils of the particular terrain which he is investigating (Bateson, 1972, p. 153). But in this study, we use interdisciplinary in another sense: for example, the man who studies the arrangement of leaves and branches in the growth of a plant finds the formal relations between stems, leaves, buds to be analogous to the formal relations of words in a sentence. He will discover a great academic value in such studies. Those who think first of the "things" which are related (the "relata") will dismiss any analogy between linguistic grammar and the anatomical 
structure of plants because they have no apparent similarity to each other. They will not accept any resemblance between a leaf and a noun. But, one way of interdisciplinary research is the very study of finding analogies between different things at a glance. In order to specify and step up the logical types in the invisible contexts, it is claimed that the forms of the relations between communication and the histogenesis process should be investigated. What should be imitated to reveal the formal relations is the form of the relationship structure. To conduct research against this background to investigate skill transference in art, a workshop was held in an art museum. Participants were directed to view some oil paintings and after that to copy them with pastel crayons, step by step. Visual artist Kenjiro Okazaki acted as facilitator. The details are as follows.

\subsection{Time and Place}

The workshop was held on December 24-25, 2011 in the exhibition hall and a large meeting room at the National Museum of Modern Art, Tokyo. The participants viewed the original paintings in the exhibition hall, and then moved to the large meeting room to reproduce the paintings which they viewed.

\subsection{Participants}

Fourteen adults participated (five men and nine women). The breakdown of their professions was as follows:

- one dancer

- one artist

- two researchers

- ten art students

\subsection{Target Paintings for Reproduction}

The target paintings were chosen with a focus on the historical development after the modern period, in which various stages of the creation process were reflected and observed in the style of painting; especially from the Impressionist period, through Constructivism, up to the abstract period. The selected paintings which the participants were required to reproduce in this workshop were the following five paintings:

- "Gold Necklace" by Ryuzaburo Umehara (1913)

- "Roses and a Girl" by Kaita Murayama (1917)

- "Renee, Green Harmony" by Henri Matisse (1923)

- "Water Mirror" by Yasuo Kazuki (1942)

- "Dog" by Kunitaro Suda (1950)

The selected paintings share a key feature in that the layout of the layers of colors perceived as figures and ground is crucial. For example, the paintings of 
Matisse, Kazuki and Suda have the same typical layout, where the layer of the figure was first painted, and then marginal layers, perceived as background, were painted in the next step. Therefore, these paintings are comprised of a hind layer of the figure and a fore layer of the ground. For example, in the case of the small painting by Matisse (figure 1), the dark green portrait of a figure is surrounded by a light green background, which is perceived as showing a kind of depth such as falling into a dark hole.

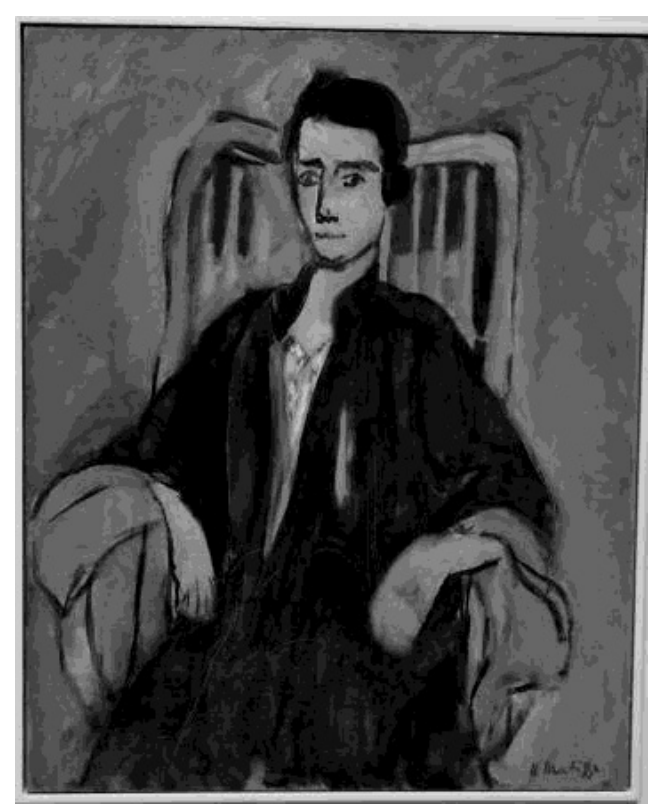

Fig. 1. "Renee, Green Harmony" by Henri Matisse (1923)

\subsection{Workshop Procedure}

The facilitator, Okazaki, named each task from A to F (Table 1). In this study, we report on the results of these tasks (the reproduction of the paintings) except for tasks A-2, A-3, and B, which involved reproducing the artworks in words. We describe the directions that Okazaki gave the participants before they viewed the paintings in the exhibition hall as "memorizing." We categorized the directions given to the participants when they returned to the meeting room after viewing the paintings and before their reproducing task as "recall." The participants were seated in rows in the meeting room so as not to give them a chance to modify their works by referring to the reproduction processes of others. A video recording was made of all the participants' behaviors. Furthermore, in order to make the painting conditions the same for all participants they received the same type and number of drawing papers and the same set of pastel crayons (Sakura Craypas, 12 colors). 


\subsection{Criteria of Analysis}

Six assessors were told to identify 14 types of behavior categories that we defined (Table 22), and they categorized the behaviors of the participants on the videos using the video analyzing software ELAN ver.4.1.2 at the maximum time resolution of the video rate. Each video scene was analyzed by two assessors.

\subsection{Results and Discussion}

Creating the Experience of Ambient Space by Using an Operating Surface Layout. The selected paintings in our workshop shared the common feature that the order of the layers between the figure and the ground is a significant key to understanding the paintings. Common sense dictates that usually the figure is regarded as being in front of the ground. But the selected paintings were created by using a reversal creation process, which was successful in inducing a strange perception. When we see the first painted surface layer in the center of the canvas, we perceive it as something like a depression, with depth. Conversely, in another painting we might perceive an ascending feeling. Using an operating surface layout creates the way the medium is perceived of the ambient space.

We are afraid to call the transition of perception induced by painting techniques the experience of beauty. To elaborate, we have doubts about whether the creation of beauty should be expected in the arts. To begin with, do artists pursue beauty? Akiba attempted to introduce Leibniz's idea of monadology to a new aesthetic apart from Kant's aesthetics (Akiba, 2011). Our question about art and beauty mentioned above corresponds with Akiba's thesis.

Kant attempted to give beauty the potential and the power to be constructed in order to overcome the problems (mutual constraints, that is, the antinomy) that are derived from each category of recognition (Kant, 1790). If this is so, however, we believe beauty gives us nothing but optimistic hopes that all will come out right, as if we are self-contained and without recognition of the truth of the world.

On the contrary, the monads are never optimistic. Following on from the philosophy of Aristotle, Leibniz postulated that sensing requires distance in time or space (that is, there is no direct perception of the object itself), and that we are surrounded by the medium (Leibniz, 1714). A simple explanation of monads is that the senses are confined by systems, but the outside is projected onto the inner perception. This means that the objects outside are understood only by a dissociation of the projected images between the inner perception and the objects outside. It was revealed in this workshop that our understanding of the perception of the object and depth, that is, the logical layers of space, was often reversed. We assume that we comprehend the very logical layers of the space (speaking in the Gibsonian viewpoint, the way of layout of surfaces); however, we can actually perceive only that one. Furthermore, what is perceived is the gap between steps of logical layers of the space which are indicated by color layers, 
Table 1. Tasks and directions

Task A

\begin{tabular}{ll}
\hline \hline Object & "Gold Necklace" by Ryuzaburo Umehara (1913) \\
& "Roses and a Girl" by Kaita Murayama (1917) \\
& "Renee, Green Harmony" by Henri Matisse (1923) \\
\hline Memorization & "View the paintings for 15 minutes. Memorize the paintings as if \\
& you are witnesses." \\
\hline Recall & "Reproduce the paintings in a limited time, six minutes for three \\
& paintings. Reduce the number of colors as much as possible. Draw \\
& with a simple combination of colors. You don't need to finalize it \\
& because these sketches are just memos." \\
\hline
\end{tabular}

Task C

\begin{tabular}{ll}
\hline \hline Object & "Water Mirror" by Yasuo Kazuki (1942) \\
Memorization & "Take 40 seconds, just a short wink of time. Go and memorize \\
& the painting very quickly, and then draw the painting in full." \\
\hline Recall & "Select one color, whichever you want. Reproduce the painting \\
& in full in your sketch. Take about 15 seconds. Draw your memo- \\
& rized impression quickly as if you are determining the layout of \\
& snapshots, and as if to fix the whole layout in one stroke and \\
& one touch. Say, 15 seconds is exaggerated, but as short a time as \\
& possible, in one minute is best." \\
& (During the reproducing process) "You can use the pastel crayons \\
& freely. Then you can paint the whole of all the pictures at the same \\
& time."
\end{tabular}

Task C2

\begin{tabular}{ll}
\hline \hline Object & "Water Mirror" by Yasuo Kazuki (1942) \\
\hline Memorization: & "One more time, you should go to see the paintings for 10 or 20 \\
& seconds. Reproduce the paintings in only two colors. Run to the \\
& hall to see the paintings and return here very quickly.In this case, \\
& three minutes for reproducing." \\
\hline Recall & tYou should not use two colors at the same time, nor use the \\
& two colors alternatively. If you begin to use the second color, you \\
& can't use the first color again. It's a two-color process like a color \\
& print. Never use the first color after you changed the color to the
\end{tabular}

Task D

\begin{tabular}{ll}
\hline \hline Object & "Dog" by Kunitaro Suda (1950) \\
\hline Memorization & "Same as the previous process. You should go to see the paintings \\
& for one minute and reproduce the paintings." \\
\hline & "Reproduce the paintings with only two colors. Just as in the \\
& previous process, if you begin to use the second color, you should \\
& not use the first color again. Yes, that way. Color over the drawing \\
& paper efficiently. You may guess the value of the two colors as if \\
& you are charged twice as much by a printer for using two colors."
\end{tabular}


Table 1. (Continued.)

Task E

\begin{tabular}{ll}
\hline \hline Object & "Renee, Green Harmony" by Henri Matisse (1923) \\
\hline Memorization & "Once more, go to see "Renee' by Matisse. The time duration is \\
& much shorter than in the previous case, 30 or 50 seconds." \\
\hline Recall & meproduce the paintings with only two colors. Follow the same \\
& which color to use first. You must meditate on which color should \\
& be first, considering that if you mistake the order of color use, you \\
& cannot repair it, resulting in crucial damage to the reproduction."
\end{tabular}

Task F

\begin{tabular}{ll}
\hline \hline Object & "Gold Necklace" by Ryuzaburo Umehara (1913) \\
\hline Memorization & "This painting's title, 'Gold Necklace', is significant. If you had to \\
& express this painting with one color, what is that color?" (In this \\
& case, the participants were required to reproduce the paintings \\
& without an opportunity to view the painting.) \\
\hline "Use just two colors. Precise sketching ability is not \\
required. Paint a simple figure of a human."
\end{tabular}

Table 2. Fourteen types of behavior categories

Behaviors without holding a crayon / Without contact with drawing paper

Pause: Without hand gesture

Movement: With hand gestures (painting gestures in air)

Looking for crayon

\begin{tabular}{l|l}
00 \\
01 \\
02
\end{tabular}

\section{Behaviors with crayon}

Drawing behaviors (Handling the crayon like a pencil / Contact with drawing paper)

Tracing: Boundary (Tracing the outlines of the objects)

Reciprocating motion: Designated object (Drawing over the paper)

Reciprocating motion: Other objects (Drawing over the paper)

Painting behaviors (Drawing with the side of the crayon, like a wiper, or using small broken-off bits of crayon / Contact with drawing paper)

Tracing: Boundary (Emphasizing the outline of the object such as brushing 4

in white around the object)

Reciprocating motion: Designated object (Drawing over the paper)

Reciprocating motion: Other objects (Drawing over the paper)

\begin{tabular}{l|l}
\hline \hline Pause behaviors (Handling a crayon Without contact with the paper) \\
\hline Without hand gestures & P0 \\
With hand gestures (painting gestures in air) & P1 \\
Other (Rubbing the paper with fingers, etc.) & 7 \\
\hline
\end{tabular}


for example in the painting by Matisse, which demonstrate the breakdown of logic, or the gap as the immeasurable depth.

The object being perceived might be like a code that we provisionally presuppose in order to accept through making that breakdown of logic fit the framework. In this meaning, the object is regarded as an aim that perception processes cannot validate if it is not regarded as it is. Its existence is neither directly perceived nor proved definitely in advance but is regarded as the aim that makes the perception and action realized, and in the midst of the perception process this aim governs or regulates action, that is, the personal standard. For example, computing is only possible when the existence of a solution as well as the solvability of a problem is ensured. To the contrary, computing is meaningless when the solution is constantly valid. The thought and the perception are not established when the provisional object (referred to as "object X"), such as solutions in computing, may be derived but has not been directly comprehended. In other words, the object $\mathrm{X}$ is comprehended as what governs the medium, as the volume that parts recognition in the perception process; that is, the gap. Depth perception is the very possibility of action and does not enclose our perception in mere subjectivity. These are referred to as open access externalities, the conditions that enable the existence of others, for that reason. We suppose that the ambient space which Gibson defined can be interpreted in this way. The object $\mathrm{X}$ is the gap that enables our perception, the medium, and the opportunity for reflection. Additionally, that is, a personal standard, which is an opportunity for speculating about actions. The object $\mathrm{X}$ has not existed yet, but is definitely possible in the future. This temporal gap, the time delay, and the volume of the medium are the surrounding space of our perception, and this is also the condition of which time consists. This provisional object $\mathrm{X}$ can be something like a hope that is cast on the world, and is returned to us in the perception process.

We don't see the object in a painting, nor is the object painted in the painting. In discussing Matisse's painting, we first operate our perceptions, capture the gap that is the very depth (overlapping layers of paints), enter in it, and try to perceive the depth consciously; we perceive the image of the woman, resulting in finding out what the image is. A mystery of the existence of time and space that bundles and enables the relation between the object and the subject who sees, lies indeed in the time delay between the time we begin to glance at the paintings unmindfully and the time we find something there. Therefore, the paintings realize (more than express) the very time and space, that is, the medium. Otherwise, the paintings cannot express the space or the objects.

To reiterate, we substantively and concretely experience a void depth (a lack) that layers of colors reproduce. When our points of view (senses) enter that lack (the hole), devote themselves to it, and cannot escape from it, we can obtain acceptance in peace by naming it as "a woman" and recognize it again. We cannot deny this accepting process is not what is referred to as the perception of beauty. However, to name it "beauty" and make it completed in the way of preestablished harmony will prevent us from understanding the essential crisis of the process. The object is both assumed and broken down, therefore perception will become possible; that is, the production of admiration. Recognizing the clues 
of the depth or the gap of the internal perception to see the paintings without assurance of the object outside leads us to rediscover and recreate the object that is the image of the woman. It is indeed a real pleasure to admire these paintings. If we live in peace with the perception of beauty, comprehending real pleasure becomes difficult.

From Drawing to Painting. The understanding process of the workshop participants who were told to reproduce the paintings after viewing them has been estimated based on the video-recorded physical motions of the participants while reproducing the artworks. At the beginning of the workshop (task A), the participants received no constraints other than of time, and they reproduced the paintings with the free use of all twelve pastel crayon colors. In that period, they reproduced the paintings using many drawing techniques. To state it concretely, most of them tried to reproduce the object by demarcating outlines on the drawing paper. This means that most of the participants subjectively comprehended what the "object" is in the original paintings. But by the time for tasks C and D, Okazaki directed the participants to use only two colors, and furthermore he told participants to use the colors one after another in order, like a two-colored print. Around this time, most of the participants drastically changed their method of reproduction from a drawing-based method to a painting-based one, and the quality of their reproduced paintings obviously improved. One example is shown in figure 2 .

Indirect Control as "Techne". In this workshop, the facilitator Okazaki did not give the participants direct-control directions: for example, "Do not use line drawing," "Do surface painting," and so on. It was found that the phase transition of behavior patterns occurred and produced the same structure of the original works when indirect-control directions were given. That is, the constraints on use of the painting tools rigidly restricted the temporal order of tool usage, and the directions functioned as boundary conditions for the participants' behaviors, leading to a desirable way of understanding the intentions of artists in order to capture the surrounding world. The distinction between direct and the indirect controls which is mentioned here comes from Deguchi's study which proposes the model of multi-subject complex systems (Deguchi, 2004). With direct control, the local constraints regulate the agent's behavior and the interactions between the agents for each. With indirect control, on the other hand, a global framework determines the global behavior of the whole system.

Giving explanations in advance about the creation process of the arts and direct specifications for the creation method seems as if it would be a more effective way to understand and create the arts. However, the results of this study suggested that indirect control is a more efficient way to provide constraints to people's unconsciousnesses than through other, direct ways. Rather than by transmitting directions directly to the consciousness, indirect control provides constraints to the unconscious self which are recognized and affirmed afterwards by the consciousness. In this way the body's behaviors can be manipulated. 


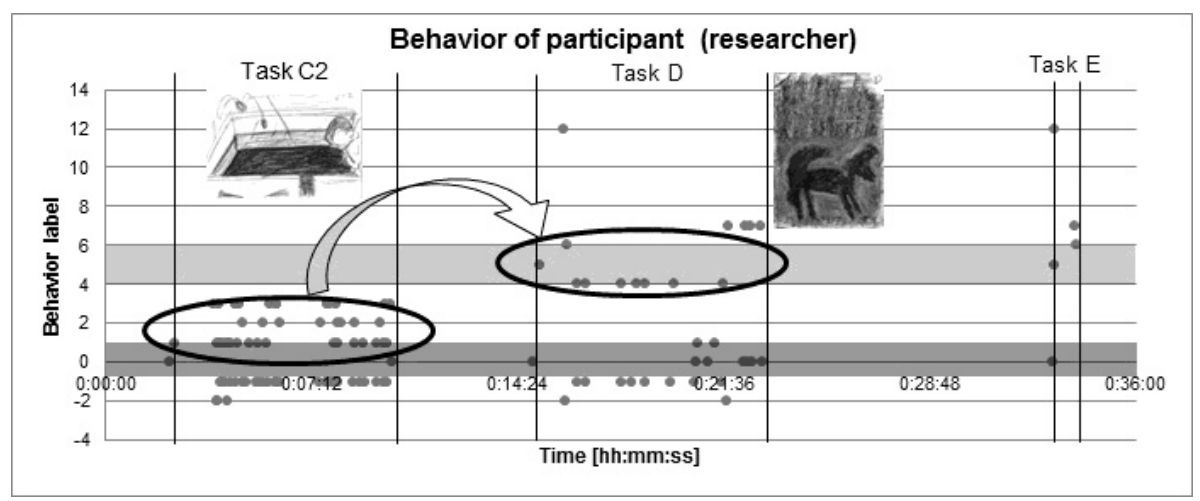

Fig. 2. Behaviors of the participants. Dark gray and light gray areas indicate drawing behaviors and painting behaviors, respectively.

Without an appeal to changing behavior at an unconscious level, directions encounter resistance from aspects such as the consciousness' self-awareness and self-respect, which does not lead to reflection on the behavior or a change in its direction.

Additionally, the reason that the people recognize indirect controls as "indirect" is that they are not aware of the body's consideration as "consideration", or regard it as involuntary. We can note that creators or those who can understand the creation process are aware that the most direct form of control in terms of skill transfer or understanding the works comes actually through an appeal to the body's consideration to indirect intelligence; in other words, direct perception. While this type of consciousness is peripheral and excluded from the point of view of the language center's functions, it is organized as a language in itself. This is in terms of "techne," the intelligence used to construct artifacts. The purpose of this workshop was to reveal empirically how "techne" is structured. If this is regarded as unconscious, it comes from suppressing consciousness that is governed by a special linguistic language.

In task B, early on between the tasks involving crayon reproductions of the paintings, the participants expressed their linguistic comprehension of the paintings (that is to say, to express the painting in words), which is not discussed here because the detailed analysis is not finished. Some participants who comprehended nothing but the outline of the object in the painting in the reproducing tasks nevertheless picked up the properties of the woman figure's depression as shown by using the word "pensive" for "Renee" by Matisse. That participant may have correctly perceived something at the unconscious level of sense, though they were not aware of the structure of paint layers on the canvas. An aim of this workshop was to specify the logical structure of ways to comprehend multilayered paintings, involving comprehension through the motions of reproduction and linguistic comprehension with free associations that seemed to have no relation to each other. In future, we have to develop more precise methods to 
investigate the invisible multi-layered logical structure without either flushing it into the black box of unconsciousness or having exaggerated feelings that we can perceive consciously.

Natural Computing and Ambient Space. So far, research on indirect control has been mainly discussed in terms of problems of contrived controls (politics, economics, drama, etc.) (Deguchi, 2004; Goan, Fukaya, 2007; Goan, Fukaya, Tsujita, 2008). Among this research, a system was modelled which acted as a dual dynamics system, in which the controller and the agents to be controlled were completely divided and there are strong interactions, which revealed the mechanism of exchanging information or of the state transitions in the system. On the other hand, the idea of contrived controls which adhere to the concept of "control," i.e. the concept that the controller should control other agents as it likes, is considerably weakened in the concept of natural computing proposed by Suzuki (Suzuki, 2012).

The following example explains this idea using the relationship between plants, harmful insects, and their natural enemies (Suzuki, Sakai, 2012). Plants (system) cannot and need not directly recognize both harmful insects and their natural enemies as objects. But the plant behaves (changes the conditions of the endocrine system) as if it can recognize insects and their natural enemies and control their behavior. What is the implementation mechanism for such plants' natural controlling behaviors? The purpose of their research is understood as clarifying the algorithm that induces state transitions in natural systems. Here we can note the possibility that the plant (system) can and may recognize the following two items, although they cannot directly recognize the existence or the behavior of harmful insects and natural enemies of the insects.

1. Recognition of the endocrine system's change: identify the trigger of the change and pay attention to the response relations.

2. Recognition of the fluctuations of the system's constancy (homeostasis): in other words, for example, perceive rhythmic change in physical conditions that get worse or become better.

A system involves these fluctuations and changes in a living process as part of its existence, even if it cannot directly recognize the objects outside the system; that is called "nature" in the concept of natural computing, and we call it "nature A." Therefore, when we assume a consciousness, the system becomes capable of being comprehended and measurable, even it is unconscious. That is like the weather condition.

Thus the ambient space can be understood as the space which enables the system to fluctuate as an adjusting process, or the field itself of which both measuring and adjusting simultaneously exist. Namely, that is the field or space itself in which a system, a species called plants in this example, can exist and be confined. In other words, the ambient space is the field that surrounds and confines the plants' intersubjective system, which is composed of surfaces and parameters 
that can be measured and extended. To make it possible for the plants to capture the critical discontinuities in the space - the change of physical conditions or the change of weather - is the very function of the ambient space. So the plants (system) become able to recognize the field on which they have dependence and in which they localize themselves, and in which they are able to exist. The field is comprised of the conditions that enable the plants' recognition, measurement, and computation of the field. Therefore the field enables the plants to measure and to compute the process of measurement or computing.

We call this field "nature X" to distinguish it from "nature A" previously mentioned. Nature X cannot be an "object" but be the "thing-in-itself." Nature $\mathrm{X}$ itself is the process of computing and recognition. It can be said that nature $\mathrm{X}$ is the recognition of the boundary between the inside (in which computing and the recursive repetition process are possible) and the outside (in which those are broken down). The preestablished harmony in recognition of the field induces no measurement or computing processes. The "repetition process" seems to repeat the same things, but actually it is not just repeating them. The significant part of the repetition process is to clarify the medium itself: the time flow whose homogeneity and continuity are never assured, in which it is possible for the computing process to induce the same solution every time. In other words, the importance of the computing process is not in the solution but in the recognition and the certainty that there are processes with no effect on the solution, and there are inevitable and irreversible distances. Not a computational solution, but the computing process itself is crucial to the medium property.

If we have an algebraic problem such as "what is one plus one?", the repetition process always induces the same solution, "two." But the short (or long) time lag for reaching the solution is the essential issue. The very time delay of this type produces the incident (or the art). This is not the issue with beauty. The concept of beauty is nothing but a convenient idea that finalizes the time flow, which is required to solve the puzzle. Due to indications that any difficult puzzle can be solved, it is easy to forget that there are valid distances, such as time flow and space.

Consider why the paintings do not consist of a unified algorithm. For example, the paintings differ from the input transfer, from the sensed information to the output, still picture. Why is this so? What does the painting of the space mean? What does the admiration of the paintings represent? What is the difference between the admiration of the paintings and the recognition of the paintings?

To address these questions, we would like to consider the following method, inspired by Aristotle's idea of entelekheia (Aristotle, 350BC): the significance of the computation (one's life) requests the computation (living) process. If we need only the results of cause and effect, the computing process may become redundant and could quickly be omitted. When the computation solutions are definite, the computation process is unnecessary. The process, the time delay, and the distance mentioned above are necessary for a slime mold, for example, but they have no meaning for observers who know the solution and regard knowing the solution as the computation. For those observers, the process of admiration 
becomes unnecessary. However, we emphasize that it is indispensable. This summarizes many of the significant issues explored in this workshop in the museum as well as Okazaki's goals (Okazaki, 2001). The concept of natural computing seems to involve the desire to avoid contrived control or the oppressive feeling from a regimented society. But even if this is so, the concept of computation tends to lead to a state that is called natural beauty, whether it is similar to that of Kantian philosophy or not. We fear that this tendency makes it difficult for us to recognize the medium property around the computation process; that is, the process itself, the distance, and the time flow. In order to avoid this tendency, we suggest that a future problem in the field of philosophy will be to bind an ethical stop standard to prevent it.

\section{From the Integration of Multiple Viewpoints to the Dissection of Multi-logical Types and Asynchronous Cooperation}

In this section, we would like to discuss the method of learning for those of us living in the ambient space. As mentioned at the beginning of this article, the incident that we call learning in this study is the step up of the logical type in the invisible meta-frame. We propose that the step up of the logical type happens when we accept that it is a dissection of the multi-logical types, deserting the idea that it is the integration of multiple viewpoints. The integration of multiple viewpoints is the idea that we cannot directly perceive the object itself, but we can reconstruct a unified image of the object using some or several numbers of partial perceptions. Namely, this means reconstruction of the unified object from multiple viewpoints. This also requires an assumption that there are some or many viewpoints to one object. As a one-to-many problem, this idea assumes the existence and the conditions of one (object X) from the beginning.

One difficulty with this idea is that it assumes the position (i.e., the unity) of the subject to the object. In this idea, however the viewpoints change, all the viewpoints are the viewpoints of the identified subject and it is tacitly assumed that their mutual continuity is assured. The position of the subject in the metalevel that integrates the multiple viewpoints is not considered to change. The viewpoints do not vary. There is no change in the relationship between the object and the subject that has an a priori assumption. Neither is there the definition nor the logical type of the space that surrounds them.

We suppose that this idea does not explain the learning mechanism. We insist that the logical type, including the relation between the subject and the object, must undergo changes or recomposition, not just a conversion of multiple viewpoints, or otherwise not only the relation between the subject and the object cannot change but also will not become stable. This is because a subject that integrates multiple viewpoints cannot avoid the infinite backward projection. The subject is always located outside the space. In other words, the subject of such a definition is not able to touch and to comprehend the ambient space that enables and ensures the relation between the subject and the object as a meta-context. 
It is natural to consider that learning occurs when the transfer and a dissection of the multi-logical types happens, not the transfer and the integration of the multiple viewpoints. To elaborate, not only are the multi-logical types dissected but they also act in parallel and asynchronously, without being unified or integrated that is, they interact, relate to each other, shuffle the domain layers of each logic, and indirectly control each other. In other words, the step up of the logical type happens when lots of dissected individual logical domains (autonomous computing circuits, i.e., the algorithms) asynchronously cooperate. We have to consider that the subject exists only in ambient space. The production process and the meta-context, the meta-level and the ambient space are comprehensive only in the acting process.

Consider the admiration of the paintings. From the traditional view of "physical space" - that the object floats in an empty space- the paintings (and the space inside) and the subjects that admire them are distinguished from each other. Until now it has been thought that this space is what accepts "the subjects who admire the paintings (and the space inside)" and surrounds them. But adopting this idea makes it seem that the subject in such a space is fated to receive the inevitable infinite backward projection as mentioned above.

However, ambient space is quite a different definition of space from the traditional one. This associates with how the paintings represent and recognize the space surrounding the subjects who admire the paintings (and the space inside) where the figure-ground reversal perception cannot exist.

Before explaining the meaning of the previous sentence, we would now like to confirm the relation between the figure and the ground in the painting. Because the figure is the projection of the observer's differentiable codes, saying it is understood as "figure-ground reversal" is not correct, but "figure-figure switch" may rather correctly express the phenomenon. The strange phenomenon which occurs is that invisible things suddenly become recognized as figures. This is just a confusion of the observer's recognition circuitry. Similar to the territorial problem, it is nothing but the difference or fluctuation between the projection of the concept and the substance onto which the concept is projected. For other examples, it is similar to asking, "Is this a lizard or a gecko?" or "Is this a duck or a rabbit?" when we see an animal for the first time. As is well known, the duck-rabbit ambiguous image occurs in the natural world, therefore, these examples are more general phenomena than the problem of the paintings.

Learning in the ambient space is not a matter of "figure-ground reversal perception" but of "figure-figure switch." The parallelizing and recomposition of the basic logic is regarded as admirable in the paintings. For example, the ground emerges at the boundary between nature and artificiality, or between fortuity and inevitability. When this relation is reversed, this is the "ground-ground switch." This concept is associated with the realization of artificial intelligence or the artificial soul. The boundary between a human that has intelligence and a material body that has no intelligence becomes invalid, and their positions reverse, like a philosopher who, in contemplation of a desk, finds himself contemplated by the 
desk. The ground-ground switch also means that we may happen to detect the critical edge behind the ground.

Here, we should note that Bateson's learning theory, the step up of the logical types, and Gibson's learning theory of perception are coupled with each other. In our workshop at the art museum, we showed that there could exist a way of perceiving the ground-ground switch, the perception of surfaces' layouts, by stepping up from the logical type of figure-ground reversal perception - the figure-figure switch. It has been said that when we observe the figure, the ground (the background as the medium) is invisible. However, this is not true. On the contrary, we found that we can even perceive the depth at the interface of the ground-ground switch through the workshop experiences and the theory. This is an astonishing discovery that shows the possibility of finding out the logical crack for dissecting the world, leading to a radical change in our understanding of the relations of whole surfaces.

1. Breakthrough happens inside ourselves; breaking our eggshell, we will hatch in another world.

2. Visual sensation without vivid focusing is the very fundamental condition that the biological organ "retina" uses to capture the optical flow. This enables our body to comprehend the space that surrounds us and continues to move on.

Acknowledgement. This work was partially supported by a Grant-in-Aid for Scientific Research (B) No. 23320049 from the Japan Society for the Promotion of Science (JSPS). We are deeply grateful to Mr. Kenji Miwa, the curator of the National Museum of Modern Art, Tokyo for his great support in implementing the workshop.

Open Access. This chapter is distributed under the terms of the Creative Commons Attribution Noncommercial License, which permits any noncommercial use, distribution, and reproduction in any medium, provided the original author(s) and source are credited.

\section{References}

Akiba, F.: Creating new aesthetics. Misuzu Shobo, Tokyo (2011)

Aristotle: De Anima (On the soul) (350 BC) (trans. by Nakahata, M.: Kyoto University Press, Kyoto (2001)

Bateson, G.: Steps to an ecology of mind. The University of Chicago Press, Chicago (1972)

Deguchi, H.: Economics as an agent-based complex system. Springer-Verlag Tokyo, Tokyo (2004)

Gibson, J.J.: The perception of the visual world. Houghton Mifflin, Boston (1950)

Gibson, J.J.: The ecological approach to visual perception. Houghton Mifflin, Boston (1979) (republished in 1986 from Lawrence Erlbaum Associates, Hillsdale) 
Goan, M., Fukaya, T.: Timing makes meaning: Protocol analysis on directions during creation in a drama-making process. In: Proc. of International Symposium on Skill Science 2007 (ISSS 2007), pp. 68-75 (2007)

Goan, M., Fukaya, T., Tsujita, K.: Two Succeeding Stages in Acquisition Process of a Rehearsed Drama: Applying System Dynamics to Human Collaborative Behavior. In: The 2nd International Conference on Knowledge Generation, Communication and Management (KGCM 2008), Proc. of The 12th World Multi-Conference on Systemics, Cybernetics and Informatics (WMSCI 2008), vol. VII, pp. 126-131 (2008)

Kant, I.: Kritik der Urteilskraft (1790) (trans. by Sakata, T.: Kawade Shobo Shinsha, Tokyo (1973))

Leibniz, G.W.: La Monadologie (1714) (trans. by Nishitani, Y., Yoneyama, M., Sasaki, Y.: Kousakusha, Tokyo (1989))

Lombardo, T.J.: The reciprocity of perceiver and environment. Lawrence Erlbaum Associates, NJ (1987)

Okazaki, K.: Inhabitants of the moon. 10+1, vol. 23. INAX Press, Tokyo (2001) (in Japanese), http://www.eris.ais.ne.jp/ ${ }^{\sim}$ ralippo/module/Study/ OKK030811_probability1/index.html

Rubin, E.: Synsoplevede Figurer. Gyldenhal, Copenhagen (1915)

Suzuki, Y.: Introduction. Abstracts of the 6th International Workshop on Natural Computing (IWNC6) (2012)

Suzuki, Y., Sakai, M.: Co-Evolution of info-chemical signal in Chemical Ecology. Abstracts of the 6th International Workshop on Natural Computing (IWNC6) (2012)

Taylor, R.P., Micolich, A.P., Jonas, D.: Using science to investigate Jackson Pollock's drip paintings. Journal of Consciousness Studies 7, 137-150 (2000) 